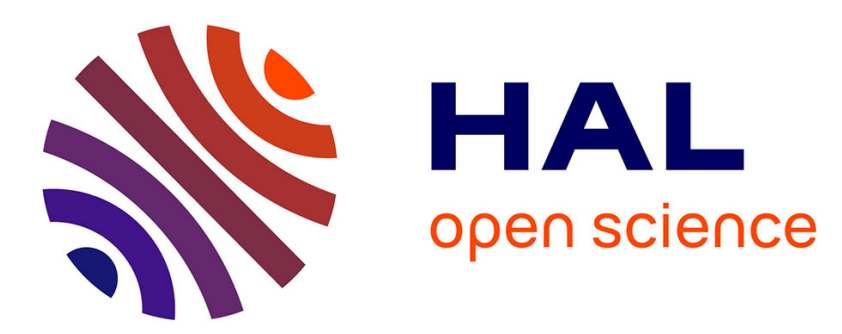

\title{
Fluid force and symmetry breaking modes of a 3D bluff body with a base cavity
}

Antoine Evrard, Olivier Cadot, V Herbert, D Ricot, R Vigneron, J Délery

\section{To cite this version:}

Antoine Evrard, Olivier Cadot, V Herbert, D Ricot, R Vigneron, et al.. Fluid force and symmetry breaking modes of a 3D bluff body with a base cavity. Journal of Fluids and Structures, 2016, 61, pp.99-114. 10.1016/j.jfluidstructs.2015.12.001 . hal-01249010

\section{HAL Id: hal-01249010 \\ https://hal-ensta-paris.archives-ouvertes.fr/hal-01249010}

Submitted on 29 Dec 2015

HAL is a multi-disciplinary open access archive for the deposit and dissemination of scientific research documents, whether they are published or not. The documents may come from teaching and research institutions in France or abroad, or from public or private research centers.
L'archive ouverte pluridisciplinaire HAL, est destinée au dépôt et à la diffusion de documents scientifiques de niveau recherche, publiés ou non, émanant des établissements d'enseignement et de recherche français ou étrangers, des laboratoires publics ou privés. 


\title{
Fluid force and symmetry breaking modes of a 3D bluff body with a base cavity
}

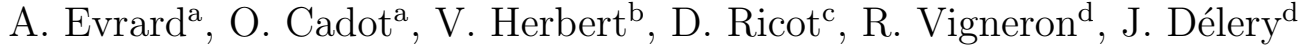 \\ ${ }^{a} I M S I A$, ENSTA-ParisTech, CNRS, CEA, EDF, Université Paris Saclay, 828 Boulevard \\ des Maréchaux, 91762 Palaiseau Cedex, France \\ ${ }^{b}$ PSA Peugeot Citron, Route de Gisy, 78140 Vlizy-Villacoublay, France \\ ${ }^{c}$ Renault SAS, 13/15 Quai Alphonse le Gallo, Boulogne-Billancourt, France \\ ${ }^{d}$ GIE S2A, 2 Avenue Volta, 78180 Montigny-le-Bretonneux, France
}

\begin{abstract}
A cavity at the base of the squareback Ahmed model at $\operatorname{Re} \simeq 4 \times 10^{5}$ is able to reduce the base suction by $18 \%$ and the drag coefficient by $9 \%$, while the geometry at the separation remains unaffected. Instantaneous pressure measurements at the body base, fluid force measurements and wake velocity measurements are investigated varying the cavity depth from 0 to $35 \%$ of the base height. Due to the reflectional symmetry of the rectangular base, there are two Reflectional Symmetry Breaking (RSB) mirror modes present in the natural wake that switch from one to the other randomly in accordance with the recent findings of Grandemange et al. (2013b). It is shown that these modes exhibit an energetic 3D static vortex system close to the base of the body. A sufficiently deep cavity is able to stabilize the wake toward a symmetry preserved wake, thus suppressing the RSB modes and leading to a weaker elliptical toric recirculation. The stabilization can be modelled with a Langevin equation. The plausible mechanism for drag reduction with the base cavity is based on the interaction of the static 3D vortex system of the RSB modes with the base and their suppression by stabilization. There are some strong evidences that this mechanism may be generalized to axisymmetric bodies with base cavity.
\end{abstract}

Keywords: Turbulent wake, Symmetry breaking, Stabilization, Drag reduction.

\footnotetext{
${ }^{*}$ Corresponding author.

Email address: cadot@ensta.fr (O. Cadot)
} 


\section{Introduction}

Bluff bodies in uniform streams are known to experience substantial form drag due to the full flow separation at their base (Schlichting and Gersten, 2000). Since they are very common in our surrounding industrial environment, such as transportation industries (ground vehicles, submerged part of ships), civil engineering (buildings, bridges) or offshore industries (risers, platforms) it is of a major interest to investigate the possible fluid force reduction that can be achieved under the constraint of conserving their functional shapes. In that context, it is useful to address the issue of reducing drag without changing the location of separation (separation control is then out of the focus of this work). Bodies with blunt trailing edge have the advantage to fix the separation and one may wonder what is possible to realize within this constraint. The response lies in the dynamics of the separated area and the low pressure therein, called the base pressure. Drag reduction mechanisms are always intimately associated with the increase of base pressure (Roshko, 1993) and their physical comprehension often points on the identification of the different contributions of the base pressure. A relevant quantity that quantifies the base pressure effect in the drag is the base suction coefficient

defined as $C_{b}=-C_{p b}$, where the pressure coefficient $C_{p b}=\frac{1}{\Sigma} \iint_{\Sigma} \frac{p_{b}-p_{0}}{\frac{1}{2} \rho U_{0}^{2}} d S$ is averaged over the surface $\Sigma$ of the blunt base. Here, $U_{0}$ and $p_{0}$ are respectively the velocity and the pressure of the uniform upstream flow.

There are several drag reduction techniques (see for instance reviews by Viswanath (1996); Choi et al. (2008)), and not all of them are yet elucidated. The passive technique that consists in producing a body cavity at the base is particularly interesting because, in addition to the fact that it leads to a substantial base drag reduction (Morel, 1979; Viswanath, 1996) in the range $10-20 \%$ for both the 2D and 3D generic axisymetric bluff bodies, it keeps unchanged the location of the separation. The effect is found to saturate for cavity depth larger than about $25 \%$ the body height. The mechanism was revealed by Kruiswyk and Dutton (1990) and Molezzi and Dutton (1995) for 2D bluff bodies. The drag reduction is related to the global Bénard von Kármán instability leading to the periodic vortex shedding. The reason for drag reduction is not an alteration of this global dynamics, but simply because of the increased distance between the body base and the zone of vortex formation. However, this explanation is not completely satisfactory, 
and the mechanism is more subtle. Actually, the pressure increase on the base reduces the drag force and then the external force necessary to hold the body. Hence the flow has to be modified. Indeed, when the drag is reduced, the vortices are found to be weakened (Molezzi and Dutton, 1995), and their pressure higher as can be clearly seen in the numerical simulation of Martin-Alcantara et al. (2014). In addition, it has to be acknowledged that at large Reynolds number flows (modeled as inviscid flow), the interaction of a flat wall with a vortex source produces an increased velocity on the wall associated with a low pressure.

Cavity effects on axisymmetric bodies are reported to give similar amounts of base drag reduction to those obtain with two dimensional bodies. On the contrary to two dimensional bodies, the reduction is observed even in the absence of wake periodicity (Viswanath, 1996). The periodic vortex shedding is then not the cause for the cavity effect. Instead, mean flow modifications are generally evoked (Viswanath, 1996) but they might be a simple consequence of the external force reduction as discussed just above. So, there is no clear interpretation of the cavity effect in this case.

The wake dynamics and topology of axisymmetric bodies are drastically different from cylinders. For instance, the first bifurcation at low Reynolds number is a steady breaking of the axial symmetry (Fabre et al., 2008; Pier, 2008; Bohorquez et al., 2011) leading to a static mode having a planar symmetry (Mittal et al., 2002), that we will refer for the remainder of the paper as Symmetry Breaking (SB) mode. As the Reynolds number increases, Berger et al. (1990) showed that the wake recovers the axisymmetry in average but presents instantaneously a large scale spatial structure that does not preserve the axisymmetry. Recent studies of Rigas et al. (2014) and Grandemange et al. (2014a) demonstrate that the planar symmetry is persistent at least for Reynolds numbers up to $2 \times 10^{5}$, but that the azimuthal position of the symmetry plane undergoes a fully random long time dynamics. The static SB modes are then present in the turbulent axisymmetric wake.

In cases of bodies with rectangular blunt base, such as the squareback Ahmed body (Ahmed et al., 1984) a similar wake dynamics has been recently evidenced. The work of Grandemange et al. (2013b) shows the permanent existence of Reflectional Symmetry Breaking (RSB) modes at least for Reynolds number up to $2.5 \times 10^{6}$. The main difference with axisymmetric bodies is that the basic symmetry of the rectangular base allows only two opposite azimuthal positions for the RSB modes. Consequently the wake dynamics is governed by a random switching between these two RSB modes 
leading to a bistable behavior. As for the SB modes of the axisymmetric bodies, the characteristic time associated with the RSB mode switching is 2 to 3 orders of magnitude larger than the time for periodic shedding with a Strouhal number $S t=0.2$ (Rigas et al., 2014; Grandemange et al., 2013b). The study of the squareback Ahmed body at low Reynolds numbers (Grandemange et al., 2012) indicates that the RSB modes are reminiscent of the two stable solutions obtained after a pitchfork bifurcation in the laminar regime. However, the closer the Ahmed body to the wall (to simulate a road effect), the larger the critical Reynolds number of the bifurcation threshold which eventually occurs in the turbulent regime (Cadot et al., 2015).

One may wonder whether the presence of the symmetry breaking modes is related to the cavity effect of 3D bodies, such as the periodic Kármán shedding is for cavity effect of 2D bodies. Sanmiguel-Rojas et al. (2011) studied the cavity effect on the stability properties of the SB modes in the laminar regime of a blunt based axisymmetric body. They found a stabilization effect, the threshold for the symmetry breaking which is obtained for $\mathrm{Re}=400$ with no cavity is postpone to an asymptotic value of about 600 for body cavities deeper than $60 \%$ of the body diameter. So, a question is how does the cavity interact with the SB modes in the turbulent regime?

The aim of the paper is to address this fundamental issue in the geometry of the squareback Ahmed body. We are aware of two studies in an industrial context from Irving Brown et al. (2010) and Grandemange et al. (2015) showing that a body cavity at the rectangular base reduces significantly the drag.

The paper is organized as follow. Section 2 describes the geometry and the measurements. The sensitivity of the wake dynamics to the body alignment in the wind tunnel is presented in Sec. 3. Results in Sec. 4 are presented in four parts. Section 4.1 characterizes the reference case (no cavity). Cavity effects are first studied on fluid forces and base pressure in Sec. 4.2 and then on the wake in Sec. 4.3. In Sec. 4.4, the cavity effect is investigated again but when a vertical control cylinder is placed in the near wake, known as a technique to stabilize the bistable behavior of the reference case (Grandemange et al., 2014b; Cadot et al., 2015). Discussions in Sec. 5 are separated in two parts. A first discussion in Sec. 5.1 aims at describing the observed stabilization within the framework of the bifurcation theory and a second discussion in Sec.5.2 interprets the physical mechanism of drag reduction using a body cavity. Section 6 concludes the paper. 


\section{Experimental set-up}

Figure 1 illustrates the set-up for the experiment. The 3D bluff body is a simplified ground vehicle similar to the squareback geometry used in Ahmed et al. (1984), but with a different aspect ratio of the rectangular base $W / H=$ 1.18 instead of 1.34 . Its dimensions $W \times H \times L$ are $350 \times 297 \times 1124 \mathrm{~mm}$. The ground clearance is set to $C=33 \mathrm{~mm}$. Also, the original cylindrical support are replaced by NACA 0025 profiles having a chord of $80 \mathrm{~mm}$. The rear of the body is equipped with a sliding base displayed as the gray board in Fig. 1(a). The sliding board dimensions are $(W-20 \mathrm{~mm}) \times(L-20 \mathrm{~mm})$. A cavity of depth $d$ is then produced by pushing the board toward the interior of the body.

The bluff body is placed in the $2 / 5$ scale wind-tunnel of GIE S2A at Montigny-le-Bretonneux especially designed for automotive aerodynamics. The facility is a closed loop circuit that is the copy at scale $2 / 5$ of the full scale facility whose detailed description may be found in Waudby-Smith et al. (2004). The plenum of the facility is sketched in Fig. 1(b), The incoming flow enters the plenum from an area contraction ratio of 6 with a 3/4 open jet having a cross-section of $3.84 \mathrm{~m}^{2}(2,6 \times 1,47 \mathrm{~m})$. The jet is then only guided by the floor. A boundary layer suction is applied in the gray area in Fig. 1(b) in order to obtain a $3 \mathrm{~mm}$ boundary layer displacement thickness $1.4 \mathrm{~m}$ upstream the centre of the body. The bluff body is placed on a large turntable having $2.8 \mathrm{~m}$ in diameter to reproduce sideslip situations with a yaw angle $\beta$. The flow inhomogeneity is less than $0.5 \%$ with an angular deviation smaller than $0.25^{\circ}$ in both the $y=0$ and $z=0$ planes. The free stream turbulent level is lower than $0.4 \%$. The maximum wind speed is $75 \mathrm{~m} . \mathrm{s}^{-1}$ but for the present study, only the results for $U_{0}=20 \mathrm{~m} . \mathrm{s}^{-1}$ are shown since investigations at larger velocities was giving comparable results. Thus, the Reynolds number of the flow is $\operatorname{Re}=\frac{U_{0} H}{\nu} \simeq 4 \times 10^{5}$.

The measurement systems are provided by the GIE S2A. The four NACA supports of the model are fixed on a 6 components balance which provides each component of the force, say $f_{i}$ where $i \in\{x, y, z\}$ denotes respectively, the drag, side and lift force component. The balance precision is $0.3 \mathrm{~N}$ for both the drag and the side force, and $0.5 \mathrm{~N}$ for the lift force. Time series are obtained from recordings at a sampling frequency of $10 \mathrm{~Hz}$ during $300 \mathrm{~s}$ when a statistically steady state is reached; part of the fluctuations are ascribed to measurement uncertainty but as we will see, they still give quantitative information on the steadiness of the forces. 

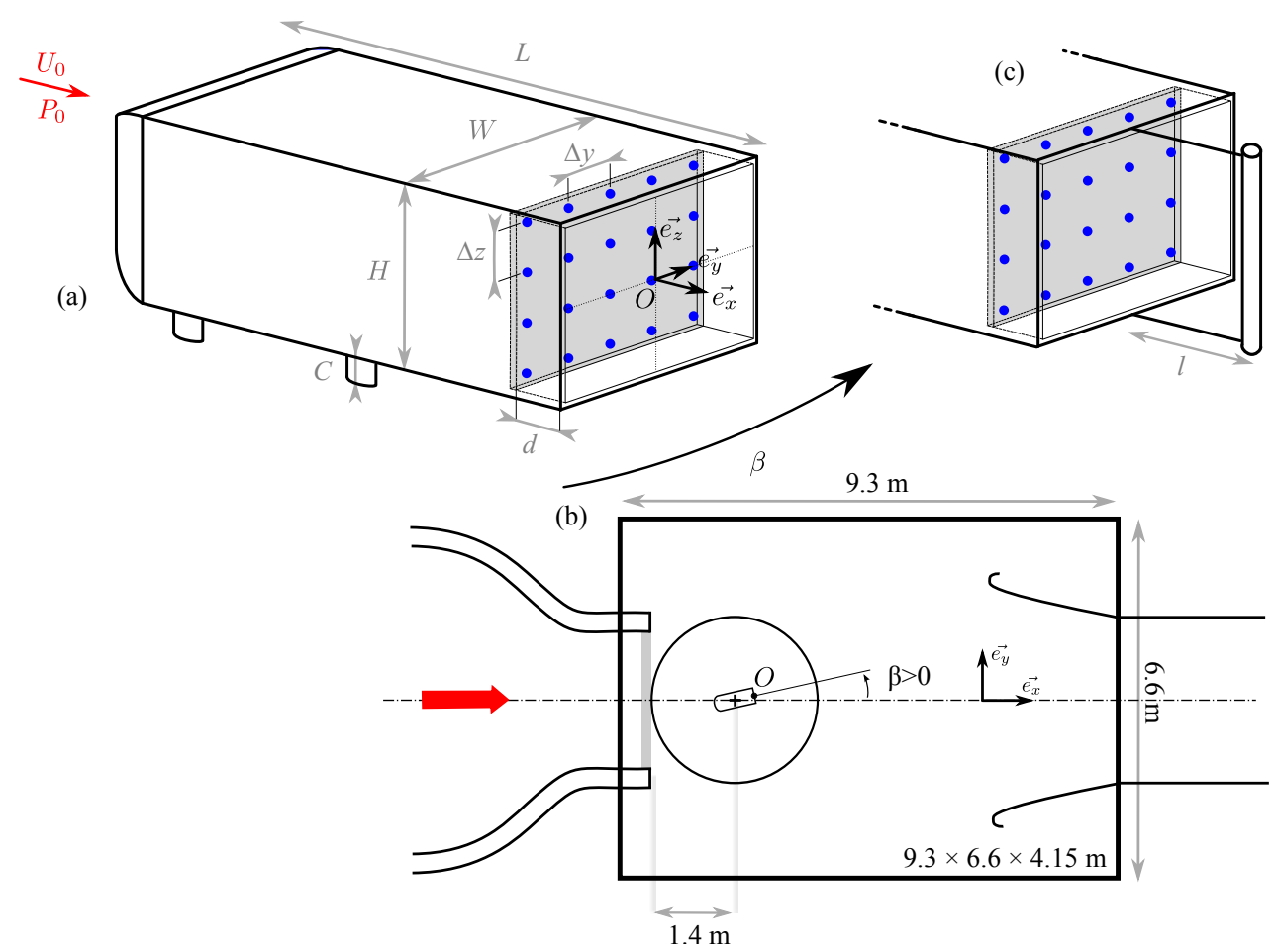

Figure 1: Sketch of the experimental set-up. Model (a) with adjustable cavity depth $d$, see text for dimensions. Blue dots represent the distribution of the pressure taps. The origin of the coordinate system is set at the centre of the body base. Plenum (b) of the wind tunnel. In (c) a vertical cylinder of diameter $0.067 \mathrm{H}$ is fixed at the rear of the body with two thin rods at a distance $l=0.57 \mathrm{H}$. The length of the cylinder is $1.064 H$.

The pressure is measured at 20 locations at the body base (indicated with blue dots in Fig. 1(a). There are 5 columns separated by a distance $\Delta y=72 \mathrm{~mm}$ of 4 pressure taps separated by a distance $\Delta z=80 \mathrm{~mm}$. The taps are distributed symmetrically referring to the planes $y=0$ and $z=0$, corresponding respectively to the mid-width and mid-height of the body. The pressure is obtained using a ZOC22 pressure scanner and a GLE/SmartZOC100 electronic for data acquisition through an ethernet connection to a PC. It is acquired at a sample rate of $200 \mathrm{~Hz}$ per channel, with an accuracy of $\pm 3.75 \mathrm{~Pa}$. The pressure scanner is located inside the model. It is linked to each tap with less than $300 \mathrm{~mm}$ of vinyl tubes to limit the filtering effect of the tubing. The 20 pressure channels are recorded during $600 \mathrm{~s}$ for typical 
experiments. The force and the pressure coefficients are defined as :

$$
c_{i}=\frac{f_{i}}{\frac{1}{2} \rho S U_{0}^{2}} \quad ; \quad c_{p}=\frac{p-p_{0}}{\frac{1}{2} \rho U_{0}^{2}},
$$

with $i$ referring to the force component, $\rho$ is the air density, $S=H \times W$ is the frontal area of the Ahmed body and $p_{0}$ is the free stream pressure. The coefficients are given to be accurate in the absolute range \pm 0.002 . Concerning the pressure distribution at the base of the body, some quantities are of major interest. The base suction coefficient is defined as :

$$
c_{b}(t)=-\frac{1}{20} \sum_{i=1}^{5} \sum_{j=1}^{4} c_{p}\left(y_{i}, z_{j}, t\right),
$$

where $i, j$ are referring to the pressure taps location at the base. The minus sign insures $c_{b}(t)$ to evolve in the same manner as the pressure drag. The two following quantities will give global information about the instantaneous wake orientation in the $y$ and $z$ direction. They are the vertical pressure coefficient profile taken at the centre of the base $(y=0)$ and the horizontal pressure coefficient profile taken at the second row from the bottom $(z=-0.138 H)$ :

$$
c_{p}(y, t)=c_{p}(y,-0.138 H, t) \quad ; \quad c_{p}(z, t)=c_{p}(0, z, t) .
$$

We define the pressure gradient at the centre of each of the profiles in the $y$ or $z$ directions as :

$$
\frac{\partial c_{p}(t)}{\partial y}=\left.\frac{\partial c_{p}(y, t)}{\partial y}\right|_{y=0} \quad ; \quad \frac{\partial c_{p}(t)}{\partial z}=\left.\frac{\partial c_{p}(z, t)}{\partial z}\right|_{z=0} .
$$

The PIV system is a dual pulse laser (Nd:YAG, $2 \times 200 \mathrm{~mJ}, 4 \mathrm{~ns})$ combined with a Dantec CCD camera (FlowSense EO, $4 \mathrm{Mpx}$ ). The setup acquires image pairs at a rate of $4 \mathrm{~Hz}$. The interrogation window size is $32 \times 32$ pixels with an overlap of $50 \%$. Conventional notations for the velocity components will be used, say $(u, v, w)$ in the coordinate system $\left(\vec{e}_{x}, \vec{e}_{y}, \vec{e}_{z}\right)$. Two measurement planes are investigated at the rear of the body; the plane $y=0$ that gives access to $u$ and $w$ velocity components and the plane $z=0$ that gives access to $u$ and $v$ velocity components. The thickness of the laser light sheet is about $5 \mathrm{~mm}$. The interrogation window of $32 \times 32$ pixels corresponds 

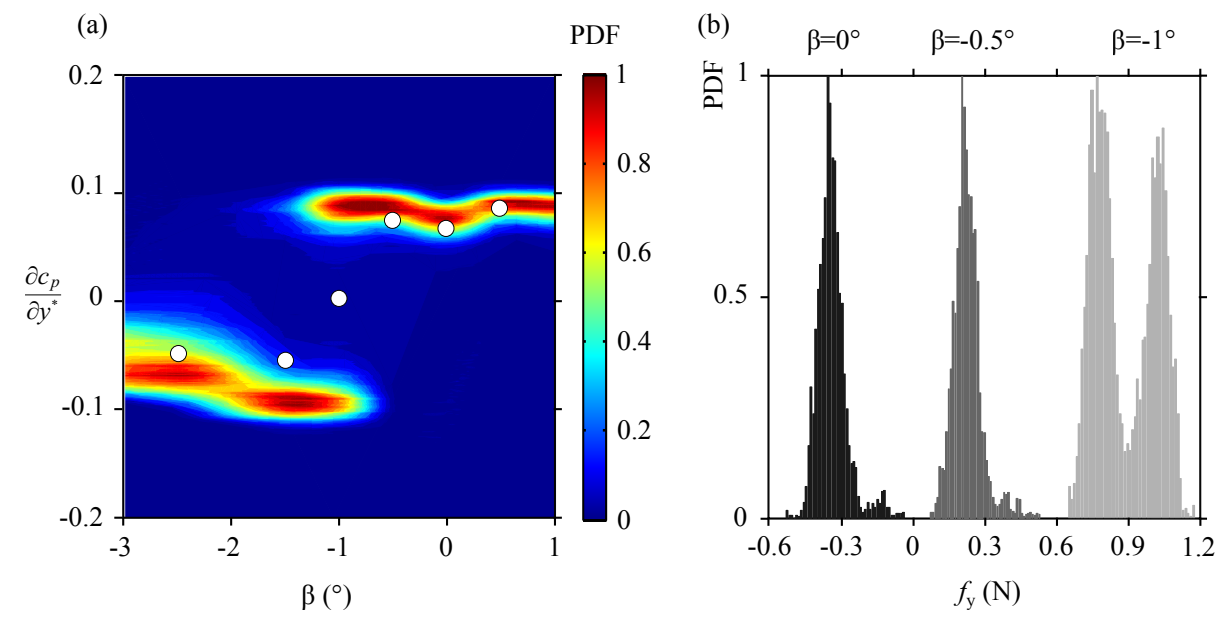

Figure 2: Probability density function (PDF) vs. the yaw angle $\beta$ normalized by their maximum value. (a): PDF of the horizontal pressure gradient at the base $\frac{\partial c_{p}}{\partial y^{*}}$, the empty circles displays the corresponding time averaged value $\frac{\partial C_{p}}{\partial y^{*}}$. (b): PDF of the side force $f_{y}$ for 3 different yaw angles.

to physical sizes of $5 \times 5 \mathrm{~mm}$. For each cavity depth $d$, 400 PIV fields are acquired in each plane to perform the statistics.

The instantaneous reflectional symmetry of the wake will be characterized by the spaced averaged $v$-component of the velocity in the plane $z=0$ defined as :

$$
\langle v\rangle_{w}=\frac{2}{H^{2}} \int_{x=H / 2}^{H} \int_{y=-H / 2}^{+H / 2} v d x d y .
$$

$\langle v\rangle_{w}$ can be seen as the instantaneous global side velocity of the near wake.

For the remainder of the paper, $a^{*}$ denotes the non-dimensional value of any quantity $a(x, y, z, t)$ made dimensionless by a combination of the height $H$ and the inlet velocity $U_{0}$. Furthermore, any time averaged quantities are denoted with a upper case letter: $A=\bar{a}$, and the prime denotes the root mean square about the mean $A^{\prime}=\sqrt{\overline{(a-A)^{2}}}$. The quantity $A$ will be called the mean of $a$ and $A^{\prime}$ its fluctuation. 


\section{Body alignment, RSB modes, bistability and fluid force}

As already pointed out by the previous studies of Grandemange et al. (2013a,b, 2014b, 2015), this flow is highly sensitive to any symmetry defects comprising both main flow inhomogeneities and geometrical imperfections. This sensitivity can be characterized by changing accurately the yaw angle $\beta$ of the model. We first show in Fig. 2(a), the variation with $\beta$ of the statistics of the horizontal pressure gradient as defined in Eq. (3). There is a clear discontinuity with two opposite most probable pressure gradients at $\beta=-1^{\circ}$. It is now admitted from these previous works that their existence is due to the two mirror RSB modes of the turbulent wake.

The left to right reflectional symmetry of the flow (with respect to the $y=0$ plane) is not respected when $\beta=0$ (corresponding to the geometrical alignment in the wind tunnel), since the wake is locked on one of the two RSB modes producing a significant positive horizontal mean pressure gradient at the base. A misalignment close to $\beta=-1^{\circ}$ is needed to obtain the equal exploration of the two RSB modes that restores the symmetry in a statistical sense (indicated by the absence of a mean horizontal pressure gradient).

We turn now to the statistics of the side force in Fig. 2(b). The force is not zero for $\beta=0$ thus confirming a small angular deviation of the main flow (it was measured to be smaller than $0.25^{\circ}$ in free stream conditions). Even small, the flow deviation is enough to lock the wake on the RSB mode having the positive horizontal pressure gradient at the base. In consequence, the value of the lateral force which is about $F_{y} \sim-0.32 \mathrm{~N}$ is a cumulative effect of a small main flow deviation together with the selection of a RSB mode. It is likely that the condition $\beta=-1^{\circ}$ is the best alignment with the main flow, because the two RSB modes are clearly observable in both the lateral force and the pressure gradient. The fact that the mean value is $F_{y} \sim 0.9 \mathrm{~N}$ is a simple consequence of the non zero yaw angle that produces a small drag force projection in the $y$ direction of the force balance measurements.

Finally, the symmetrical defect should not be ascribed to the main flow only. Indeed, in the present case the pressure scanner cable exits the model just behind the left rear support and is fixed on the floor using adhesive tape. We did a test with the cable exiting the model from the right rear support, and the best alignment was $\beta=-0.5^{\circ}$. Thus, any geometrical defects of the model will also have their significant influence.

For all the experiments presented next, the body alignment is $\beta=-1^{\circ}$ to guarantee the equal exploration of both RSB modes. 


\section{Results}

\subsection{Reference case}

Table 1: Mean and fluctuation of the coefficients $C_{i}^{\text {ref }}$ and $C_{i}^{\text {ref }}{ }^{\prime}$ (see text) for the reference squareback model with no cavity, $d^{*}=0$.

\begin{tabular}{c|ccccccc}
$d^{*}$ & $C_{b}^{\text {ref }}$ & $C_{x}^{\text {ref }}$ & $C_{y}^{\text {ref }}$ & $C_{z}^{\text {ref }}$ & $C_{x}^{\text {ref }^{\prime}}$ & $C_{y}^{\text {ref }^{\prime}}$ & $C_{z}^{\text {ref }^{\prime}}$ \\
\hline 0 & 0.149 & 0.288 & 0.037 & -0.117 & $4 \times 10^{-3}$ & $5 \times 10^{-3}$ & $4 \times 10^{-3}$
\end{tabular}

The force coefficients $C_{i}^{\text {ref }}$ of the reference case (i.e. $d^{*}=0$, no cavity) are given in Table 1 . The base suction represents $51.7 \%$ of the total mean drag coefficient $C_{x}$ that takes into account both the form and friction drag. This important contribution is a well known feature of the Ahmed body (Ahmed et al., 1984) which gives a prior interest to the flow dynamics at the body base. The body develops a small negative lift ascribed to a flow acceleration in the ground clearance. The small positive side force coefficient is a consequence of the body alignment as discussed above. The fluctuation of the side force coefficient is the largest of the three components of the force which is explained by the bistable behavior of the wake.

The bistability is the dynamics of the random exploration of both RSB modes which is unambiguously observable in the horizontal pressure distribution (defined in Eq. 2) at the base shown as space time diagram $c_{p}\left(y^{*}, t\right)$ in the left column of Fig. 3(a). The total time duration of these samples is $T^{*}=4000$. Note that the typical time $\tau^{*}$ for vortex shedding with a Strouhal number $\mathrm{St}=0.2$ is $\tau^{*}=1 / \mathrm{St}=5$. The trace of the $\mathrm{RSB}$ modes are easily detectable by the permanent strong asymmetry in the pressure distribution with one low pressure side at a level $c_{p} \sim-0.2$ appearing in blue and a high pressure side at a level $c_{p} \sim-0.1$ appearing in red.

It is convenient to identify the mode by the sign of their horizontal base pressure gradient at the centre of the base. In Fig. 3(a, left), a time interval spent in the RSB mode with negative base pressure gradient is indicated by the letter $N$ and in the RSB mode with positive pressure by the letter $P$. Eight switches between the two RSB modes are observable in (a). The time spent in one mode varies between 200 to 1000, this long time dynamics was already mentioned and its statistics thoroughly studied in the previous paper by Grandemange et al. (2013b). 

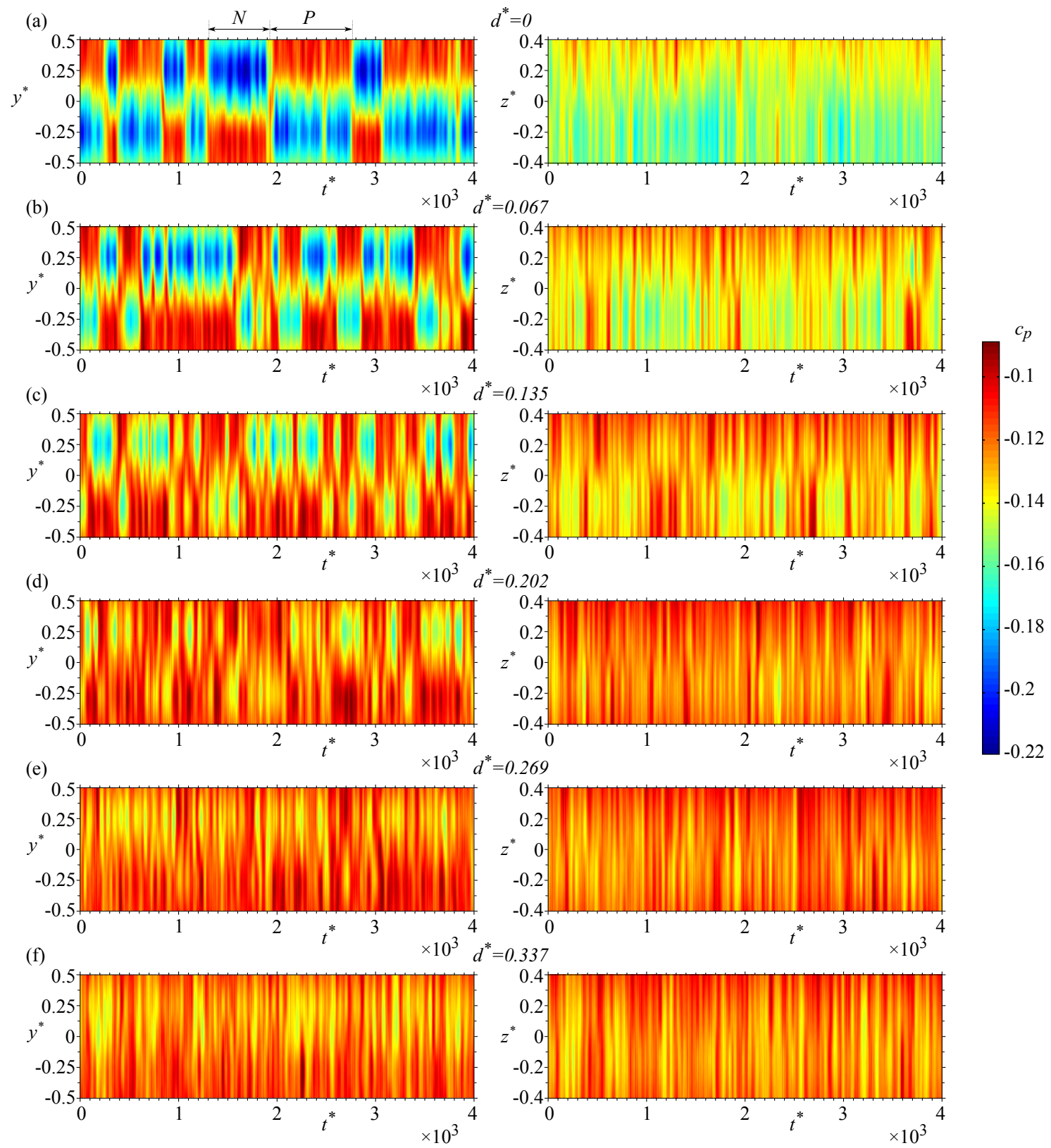

Figure 3: Space time diagram of the horizontal, $c_{p}\left(y^{*}, t\right)$ (left) and vertical, $c_{p}\left(z^{*}, t\right)$ (right) pressure distribution at the base (Eq. 2) vs. the cavity depth $d^{*} . N$ and $P$ denote respectively a time duration spent in the RSB mode with negative and positive horizontal pressure gradient. 
The acquisition rate of the pressure scanner allows to follow the dynamics of the base pressure gradient in correlation to the base suction. We show in Fig. 4(a,b) trajectories in the plane base pressure gradient versus base suction over the same time duration as in Fig. 3(a). For the horizontal base pressure gradient (Fig. 4a), the 8 trajectories linking the two basins of attraction of each mode are representative of the switching dynamics. The lowest base suction is observed during switchings having base pressure gradient close to zero.

Conditional statistics are used to obtain the property of one mode only. Since both modes are mirror from each other we will only display the mode $N$. It consists in keeping the events of negative pressure gradient only as illustrated in Fig. 5. The resulting base pressure distribution of the mode $N$ is shown in Fig. 6(a). The velocity field in the $z=0$ plane of the wake mode $N$, shown in Fig. $7(\mathrm{a}, \mathrm{c})$, is obtained by keeping PIV fields having a negative instantaneous global side velocity in the near wake (see Fig. 5). The correlation between simultaneous measurements of both the pressure and global side velocity in Fig. 5 is excellent indicating that the RSB mode $N$ is directly associated with a negative $v$-component velocity inside the recirculating bubble.

The RSB mode $N$ wake in the plane $z=0$ in Fig. 7(a) and (c) is in agreement with Grandemange et al. (2013b), the striking feature is the presence of a circular recirculation, close to the base and on the hand side $y^{*}>0$ corresponding to the low pressure footprint in Fig. 6(a). By looking at the velocity fluctuation in Fig. 7(c), we see that this circular recirculation is located in a place with low fluctuations, on the contrary to the opposite elliptical recirculation that concentrates high fluctuation level. Then, the circular recirculation looks rather like a steady vortex whose intensity is likely to be responsible for the low pressure footprint of the RSB mode in Fig. 6(a). This experimental result is confirmed by the recent large eddy simulation of Pasquetti and Peres (2015) showing unambiguously the mode N pressure field of the squareback Ahmed body at $\mathrm{Re}=5.12 \times 10^{4}$.

The RSB modes are not distinguishable neither for velocity fields in the plane $y=0$, nor in the vertical pressure gradient displayed in Fig. 3(a, right). There is then no justification to perform conditional statistics and we show in Fig. 8(a,c) the complete average of the PIV data measured in the plane $y=0$ that should be relevant for both RSB modes $N$ and $P$. 

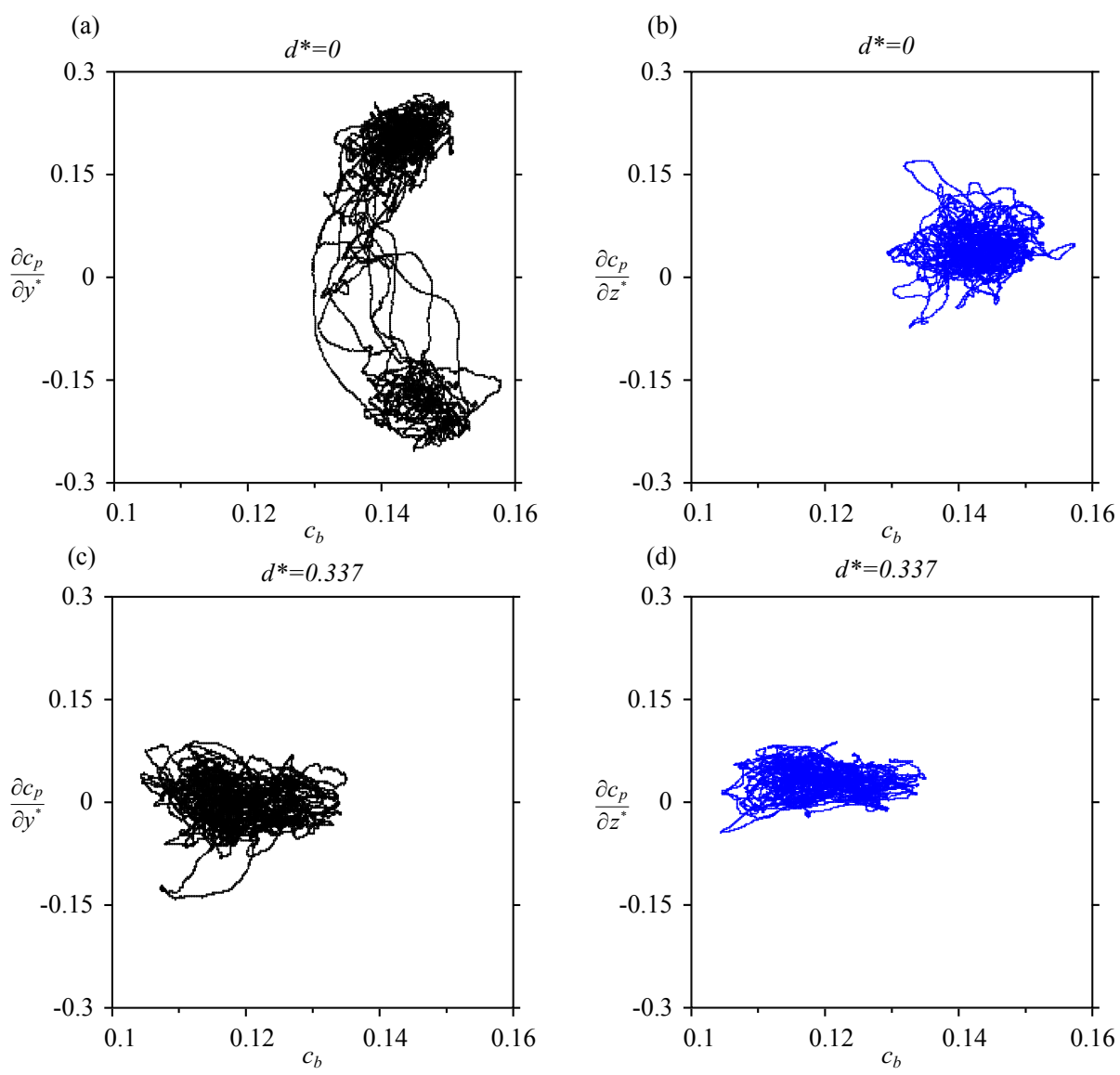

Figure 4: Trajectories in the phase diagram : horizontal base pressure gradient vs. base suction $(\mathrm{a}, \mathrm{c})$ in black curves and vertical base pressure gradient vs. base suction $(\mathrm{b}, \mathrm{d})$ in blue curves. Reference case in (a) and (b). Case with the deepest cavity in (c) and (d). 


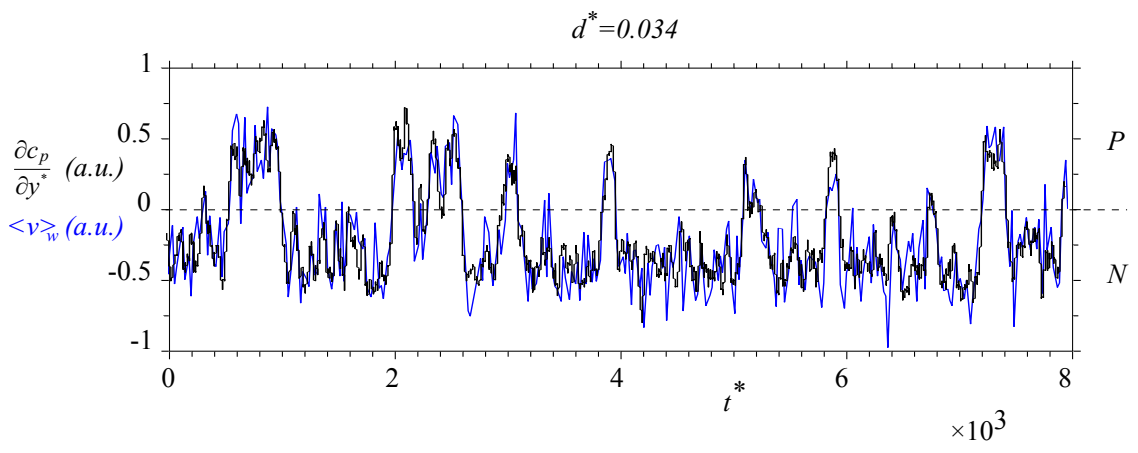

Figure 5: Signals in arbitrary units used for conditional statistics to extract events of the RSB mode $N$. For base pressure distribution, the RSB mode $N$ corresponds by definition to events of negative horizontal pressure gradient, $\frac{\partial c_{p}}{\partial y^{*}}<0$. For the wake velocity in the horizontal plane $z=0$ measured with the PIV, the RSB mode $N$ appears as negative global side velocity $\langle v\rangle_{w}<0$ of the near wake as defined in Eq. 4 . These pressure/PIV simultaneous measurements are performed for a cavity depth of $d^{*}=0.034$.

(a)

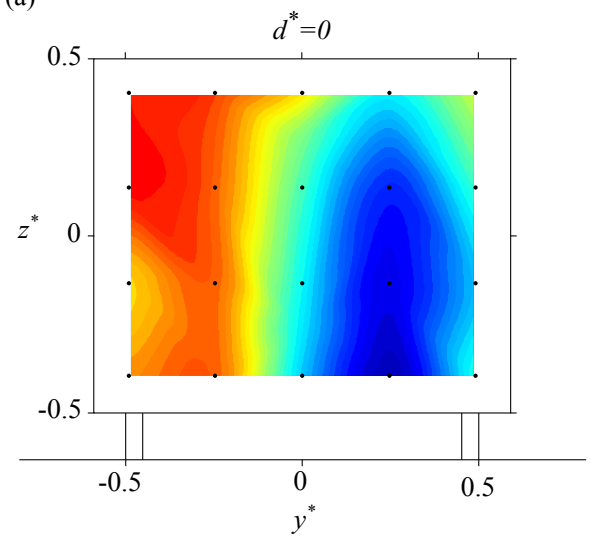

(b)

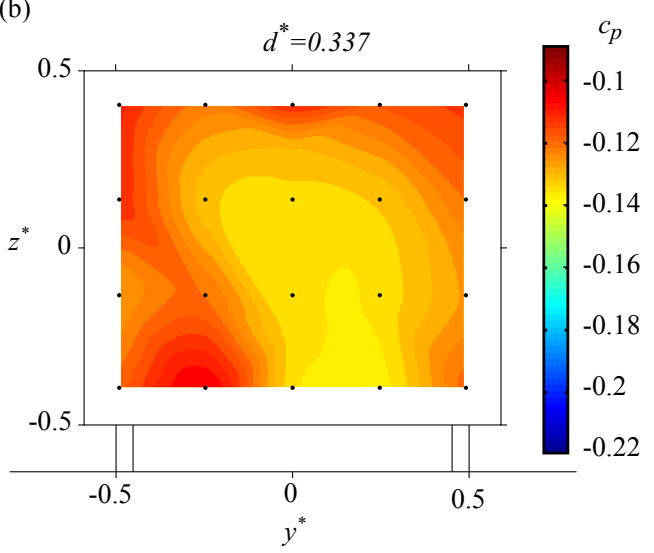

Figure 6: Mean pressure distribution, $C_{p}\left(y^{*}, z^{*}\right)$ for the reference case $d^{*}=0$ of the RSB mode $N$ (a) and for the deepest cavity $d^{*}=0.337$. 

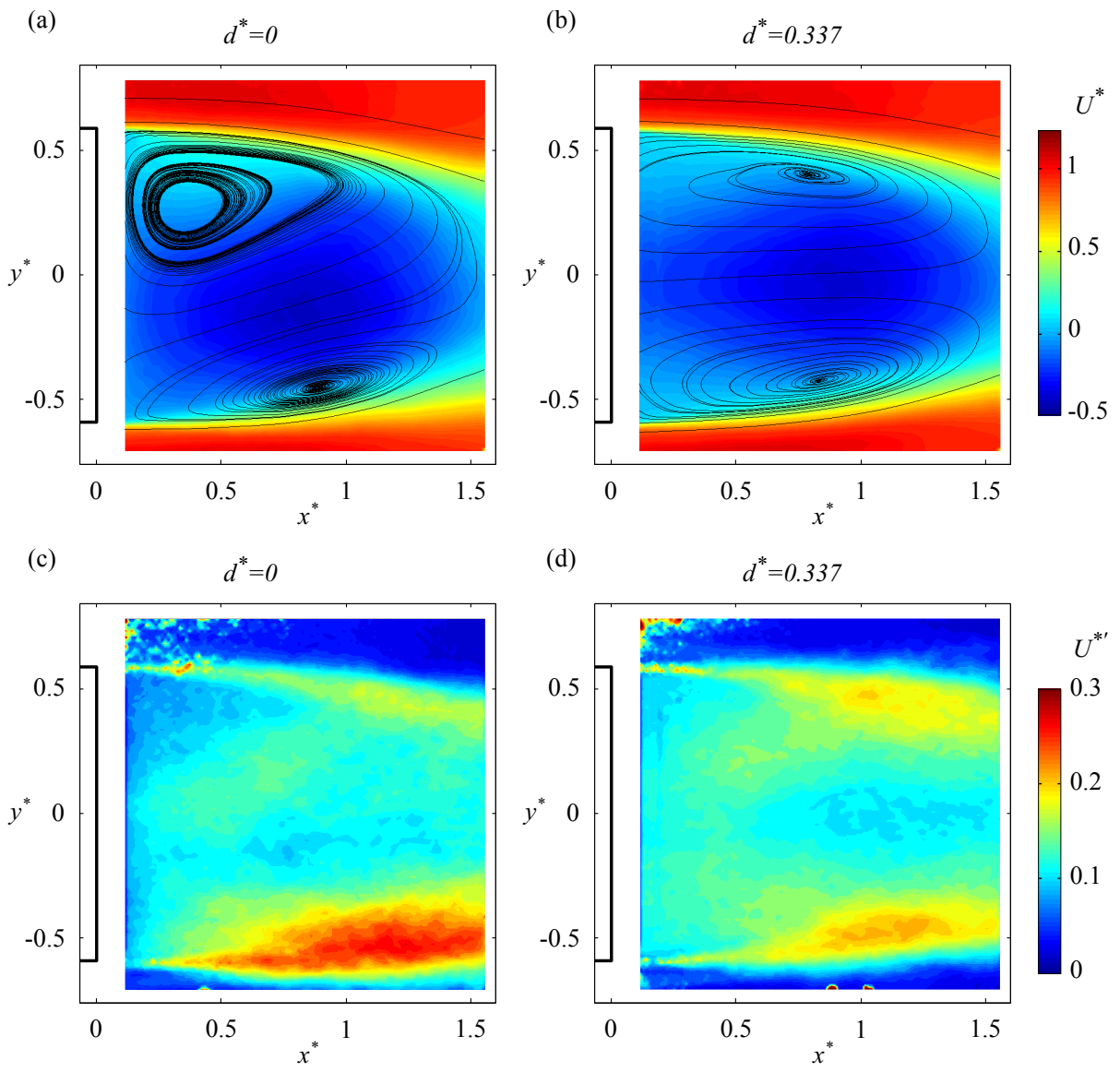

Figure 7: PIV measurements in the $z=0$ plane for the reference case $d^{*}=0$ and the deepest cavity $d^{*}=0.337$. For $d^{*}=0$, mean $U^{*}$ (a) and fluctuation $U^{* \prime}$ (c) of the $u^{*}$ component of the velocity for the RSB mode $N$ (see text). For $d^{*}=0.337$, mean $U^{*}$ (b) and fluctuation $U^{* \prime}$ (d) of the $u^{*}$ component 

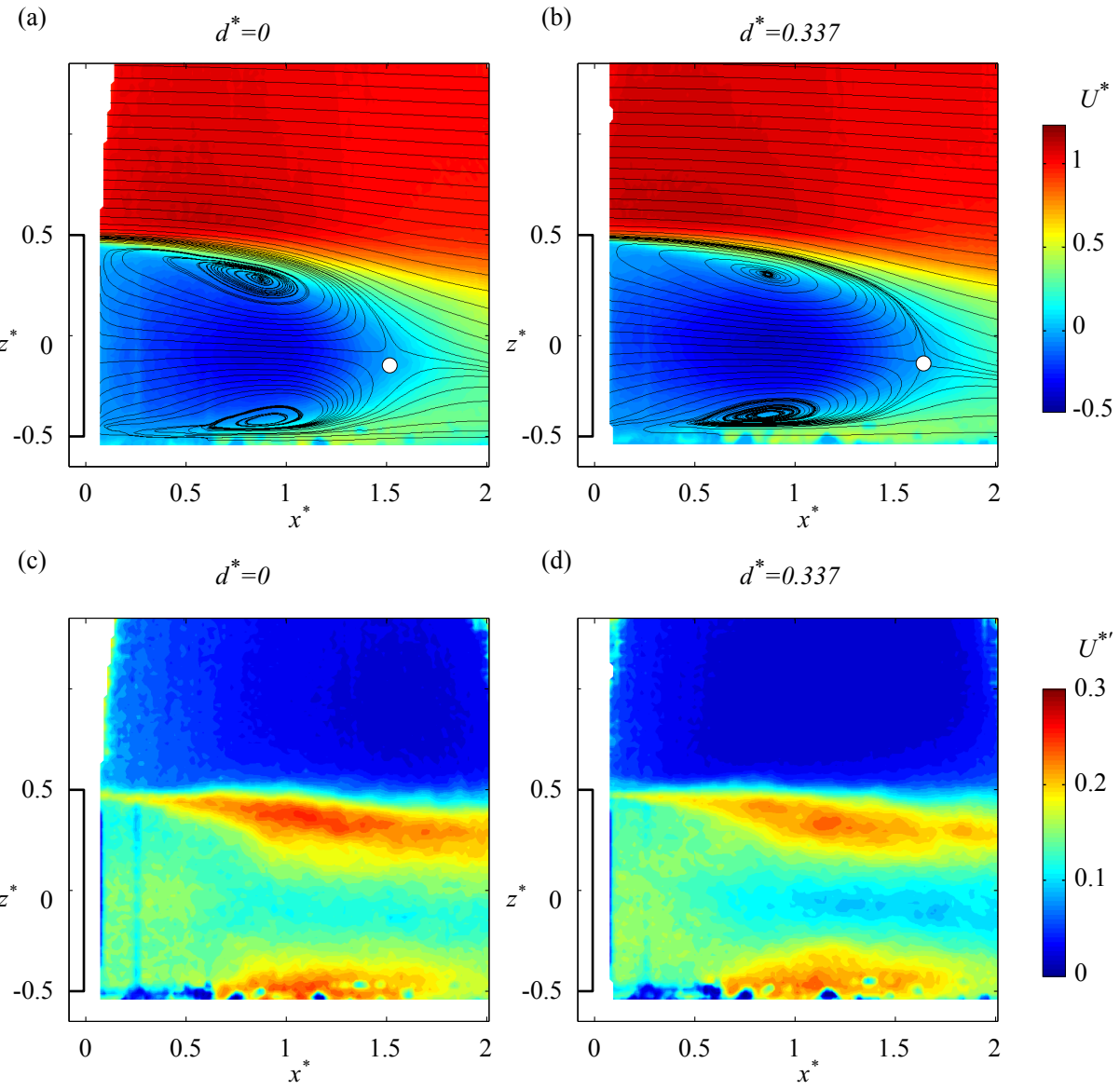

Figure 8: PIV measurements in the $y=0$ plane for the reference case $d^{*}=0$ and the deepest cavity $d^{*}=0.337$. Mean $U^{*}$ and fluctuation $U^{* \prime}$ of the $u^{*}$ component. The reattachment point in the mean flows are denoted with an empty circle. 


\subsection{Cavity effect on the fluid force and base pressure}

The evolutions of the force coefficients, $\Delta C_{i}=C_{i}-C_{i}^{\text {ref }}$, with the cavity depth are plotted in Fig. 9(a) and (b). As can be seen, a spectacular drag reduction $\Delta C_{x}$ is produced by the depth of the cavity. The effect saturates around $d^{*}=0.27$ for which the drag decrease is about $\Delta C_{x}=0.026$ corresponding to a maximum reduction of $9 \%$ from the reference case detailed in table 1 . The base suction coefficient variations $\Delta C_{b}$ are a bit more noisy but follow significantly the drag variations. It indicates that the increase of the mean base pressure is the only source of the observed drag reduction. The maximum base suction decrease can be estimated to $\Delta C_{b}=0.027 \pm 0.002$ corresponding to a relative reduction from the reference (table 1) of $18 \pm 1.5 \%$

We can see in Fig. 9(a) that the reduction does not affect either the mean lift $\Delta C_{z}$ or the mean side force coefficient, $\Delta C_{y}$. Similarly in Fig. 9(b), it does not affect either the fluctuating drag and lift but it is clearly correlated

with a reduction in the side force fluctuation $C_{y}^{\prime}$ of about $65 \%$ compared to the reference (table 1).

For each cavity depth, the pressure gradient statistics is shown in Fig. 9(c) for the vertical $z$ direction and in (d) for the horizontal $y$ direction. While no effect can be identified from the vertical gradient in Fig. 9(c), the statistics of the horizontal base pressure gradient is indicating a bifurcated diagram showing the suppression of the bi-modal behavior of the base pressure. Indeed, for a cavity deeper than $d^{*}=0.25$, the most probable value unique and close to zero, as expected from the reflectional symmetry of the set-up. The huge reduction of the horizontal pressure gradient fluctuations at the base is then responsible for the observed reduction of the side force fluctuations $C_{y}^{\prime}$ in Fig. 9(b).

The extinguishment of the bi-modal dynamics of the horizontal pressure distribution at the base, $c_{p}\left(y^{*}, t\right)$ is observable in the left column of Fig. 3. Six cavity depths from the reference case (a), $d^{*}=0$ to the deepest cavity (e), $d^{*}=0.337$ are displayed. When the cavity gets deeper, the lowest pressures (green to blue, $c_{p}<-0.16$ ) disappear, thus leaving only the level of high pressure coefficient around $c_{p} \sim-0.12$. In the $z$-direction (right column), the mode switchings are hardly detectable, and the cavity effect homogenizes the pressure distribution such that for the deepest cavity one cannot distinguish between the vertical and the horizontal pressure coefficient profiles. The same conclusion is observable in the trajectories displayed in Fig. 4(b,c) implying an isotropic dynamics of the base pressure gradients with the deepest cavity. 
(a)
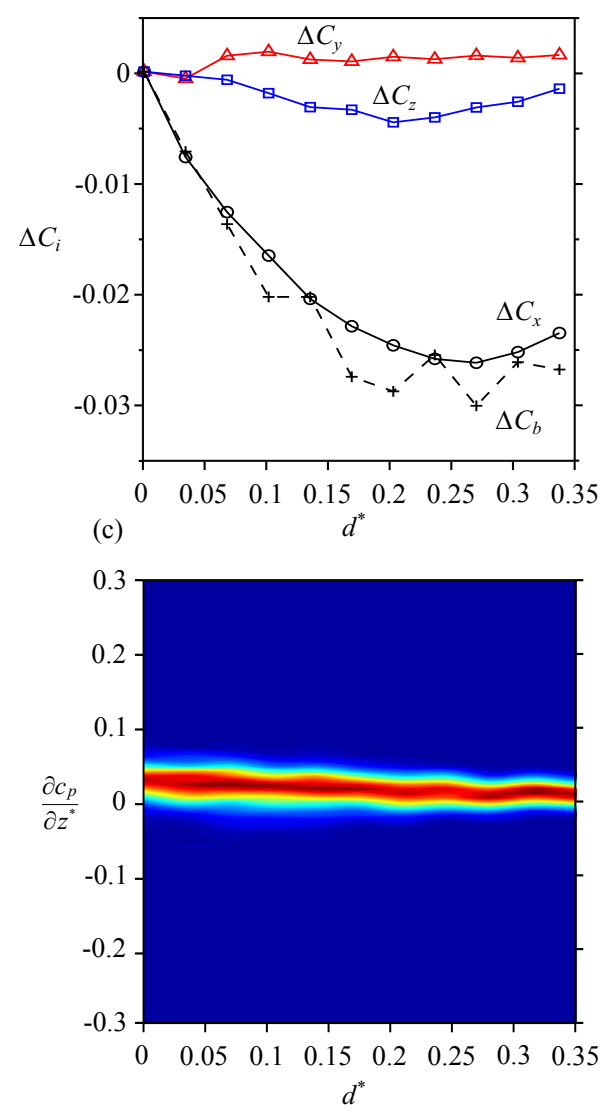

(b)
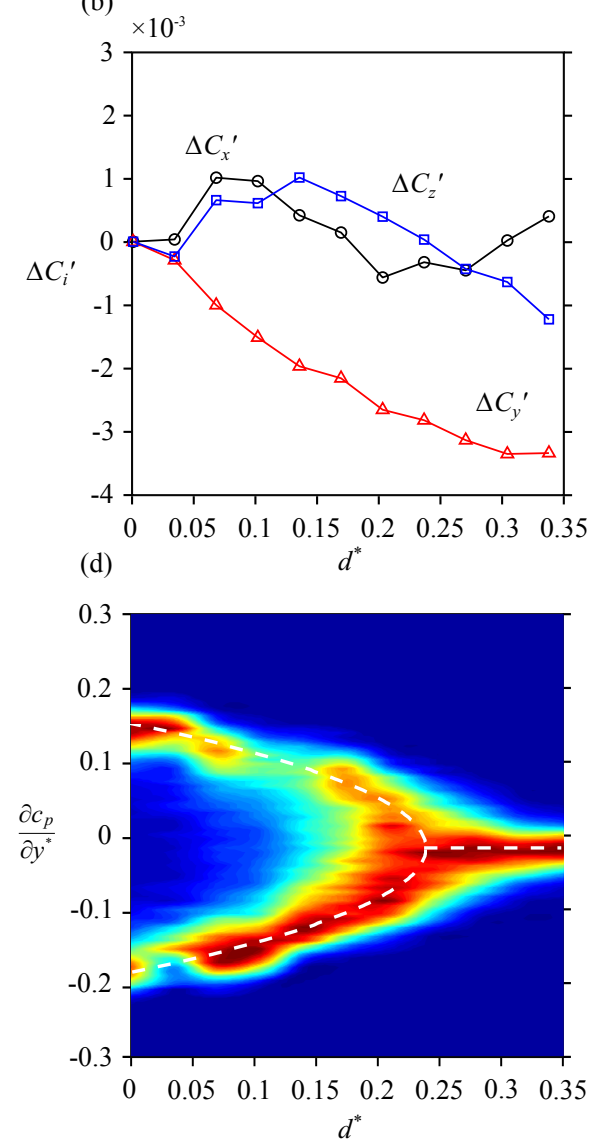

Figure 9: Variation from the reference case values given in Table 1(see text) of the mean (a) and fluctuation (b) of the force coefficients (empty symbols) as a function of the cavity depth $d^{*}$. Variation from the reference case of the mean base suction $C_{b}$ (cross symbols) in (a). Statistics of the vertical (c) and horizontal (d) base pressure gradient as defined in Eq.( 3). The PDFs are normalized by their maximum value using the same color bar as in Fig. 2. 


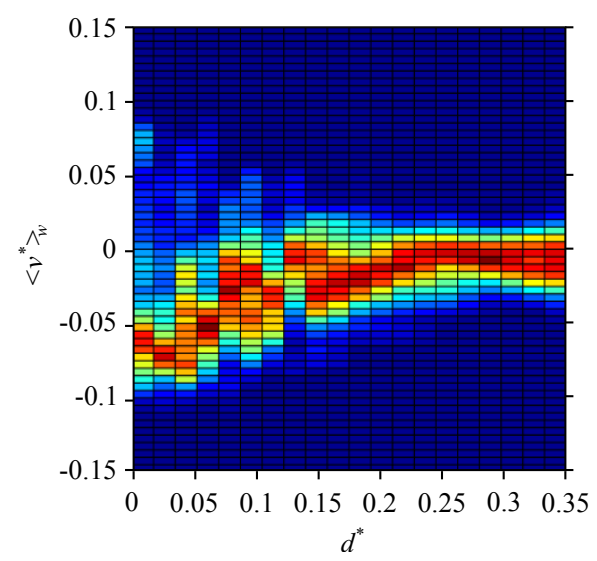

Figure 10: Statistics of the instantaneous global side velocity of the wake defined in Eq. 4 as a function of the cavity depth $d^{*}$. The PDFs are normalized by their maximum value using the same color bar as in Fig. 2.

In conclusion, the drag reduction mechanism is a consequence of the loss of the low pressure region at the base. It can be better quantified in Fig. 6 by comparing the mean base pressure distribution of the RSB mode $N$ (Fig. 6a) for $d^{*}=0$ and the mean pressure distribution for the deepest cavity (Fig. 6b) for $d^{*}=0.337$.

\subsection{Cavity effect on the wake}

The statistics of the global side velocity of the wake $\left\langle v^{*}\right\rangle_{w}$ (Eq. 4) are plotted in Fig.10 as a function of the cavity depth. The statistics are not well converged because of the only 400 realizations of the velocity fields. However they contain sufficient information to conclude about the cavity effect on the symmetry properties of the wake. For cavities such that $d^{*}<$ $d_{c}^{*}=0.24$, it is clear that during the PIV measurements, the dynamics is essentially exploring the mode having the negative side velocity, say mode $N$. For cavities deeper than $d_{c}^{*}$, the most probable side velocity of the wake is unique and close to zero as expected by the body symmetry, thus indicating a stabilization of the wake on a symmetry preserved mode. For these large deepness, the symmetry preserved mode of the wake is then characterized by the total averaging of the velocity fields as shown in Fig. 7(b,d) and Fig. 8(b,d) measured respectively in the two perpendicular planes $z=0$ and $y=0$.

In the horizontal plane $z=0$, the stabilized wake (Fig. $7 \mathrm{~b}$ ) using the deep cavity $d^{*}=0.337$ presents two symmetric elliptical recirculations. We 


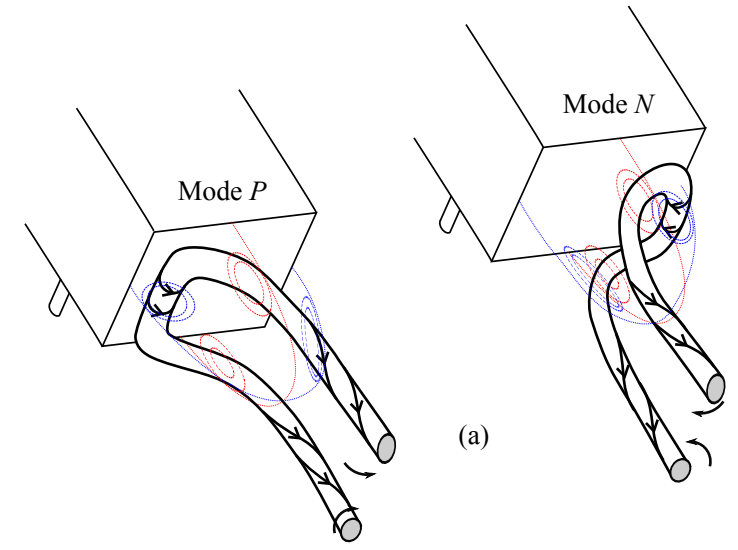

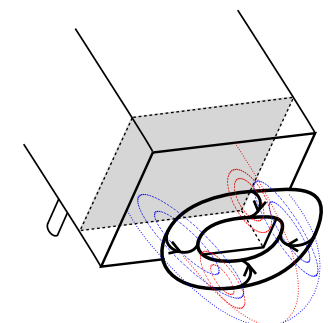

(b)

Figure 11: Schematic drawing of the mean vortex system in (a) the reference wake showing the two mirror RSB modes and in (b) with a deep cavity.

note the absence of circular recirculations comparable to the reference wake (Fig. 7a). We can also observe a global reduction of the fluctuation level in the mixing layers in Fig. 7(d) compared to the reference case in Fig. 7(c).

A comparison between the mean flows in Fig. 8(a,b) measured in the vertical plane $y=0$ reveals that the recirculating bubble length, defined by the separating distance from the body base to the bubble closure at the reattachment point, is increased by the presence of the cavity. This is a general result of the relationship between the base suction and the bubble curvature, that is well reproduced by the steady Euler flow modelling of separated flows (see general review of Wu (1972)). Since the base suction with the cavity is decreased, pressure gradients that are mainly compensating centripetal accelerations are also decreased attenuating the flow curvatures. A second observation concerns the small but significant reduction of turbulent fluctuations in the mixing layers with the presence of the cavity in Fig. 8(d) compared to the reference case of Fig. 8(c).

The main conclusion about the wake velocity measurements is that the RSB modes of the wake are suppressed for cavities deeper than $d_{c}^{*} \sim 0.24$ leading to a symmetry preserved wake. The steady vortex lying on one handside of the base is then replaced after stabilization by symmetric elliptical recirculations. It is useful to speculate a schematic view as drawn in Fig. 11 of the three dimensional mean topology of the vortex system for the two extreme cases. It is inspired from the two perpendicular measurement planes in Fig. 7(a,b) and Fig. 8(a,b). For the reference case in Fig. 11(a), the flow 
explores randomly the two RSB wakes whose mean topology is depicted by a horseshoe vortex system. With a sufficiently deep cavity, the horseshoe vortex system is replaced be a toric recirculation in Fig. 11(b) which retrieves the reflectional symmetry of the geometry. Another important point of the results concerns the turbulent fluctuation level in the wake, that is reduced by the presence of the cavity.

In the next section, we compare the effectiveness of suppressing the RSB modes with the cavity to the technique of Grandemange et al. (2014b) inspired from Strykowski and Sreenivasan (1990) to attenuate the bistable behavior by placing a small control cylinder at the centre of the recirculating bubble.

\subsection{Control cylinder versus cavity effect}

The body is now equipped with a vertical control cylinder as depicted in Fig. 1(c). The experiments are reproduced varying the cavity depth, but with shorter force and pressure time recording of $T^{*}=4000$ and without any PIV measurements. The effect of the cylinder on the mean force coefficients and base suction is shown in Fig. 12(a) in comparison to the reference case (Table 1).

We first discuss the case with no cavity $\left(d^{*}=0\right)$, the addition of the control cylinder produces an overall drag coefficient decrease of 0.011 which corresponds to a drag reduction of 3.8\% similar to the value obtained in Grandemange et al. (2014b). It is accompanied to a lift coefficient increase, with no effect on the side force coefficient. About the fluctuations in Fig. 12(b), they are significantly increased for the lift coefficient and decreased for the side force coefficient compared to the reference case. Comparing the base pressure gradient statistics at $d^{*}=0$ with the reference case in Fig. 9(c,d), we see that the cylinder actually spreads the statistics of the vertical gradient and significantly affects the statistics of the horizontal pressure gradient. There is a continuum of values within the range defined by the two most probable values of the reference case, which causes the side force fluctuation reduction. The RSB modes are then surely affected by the introduction of the control cylinder but not suppressed. Strong asymmetry reversals are indeed still observable in the space time diagram of the horizontal pressure coefficient profile in Fig. 13(a,left) but without any discrete states as confirmed by the trajectories in Fig. 14. We can notice from these phase diagrams, that the events of smallest base suction (lower drag) are correlated to the absence of base pressure gradients. 
(a)
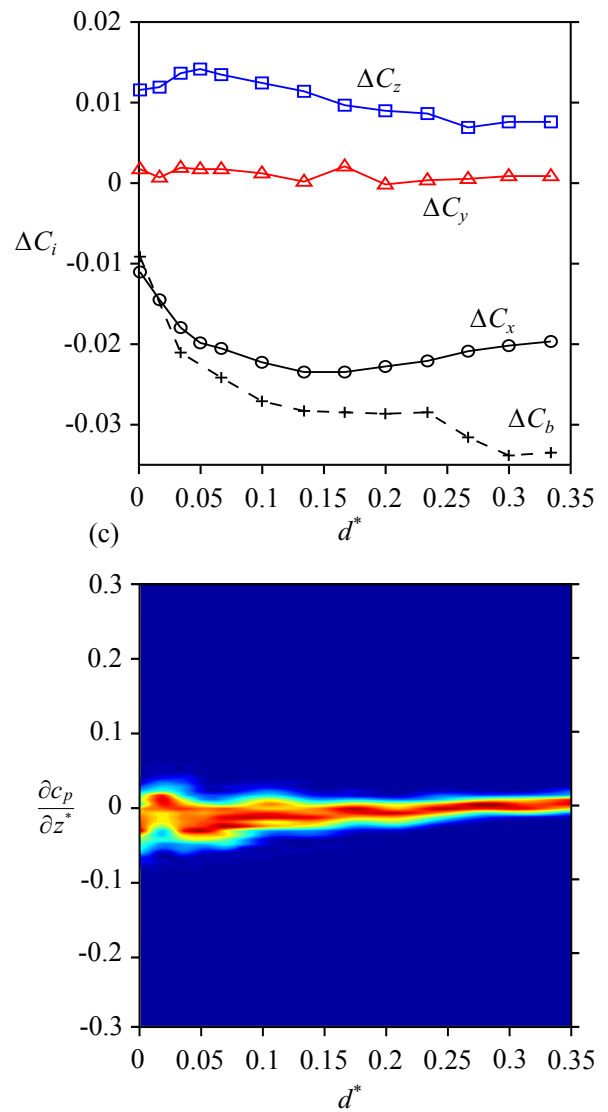

(b)
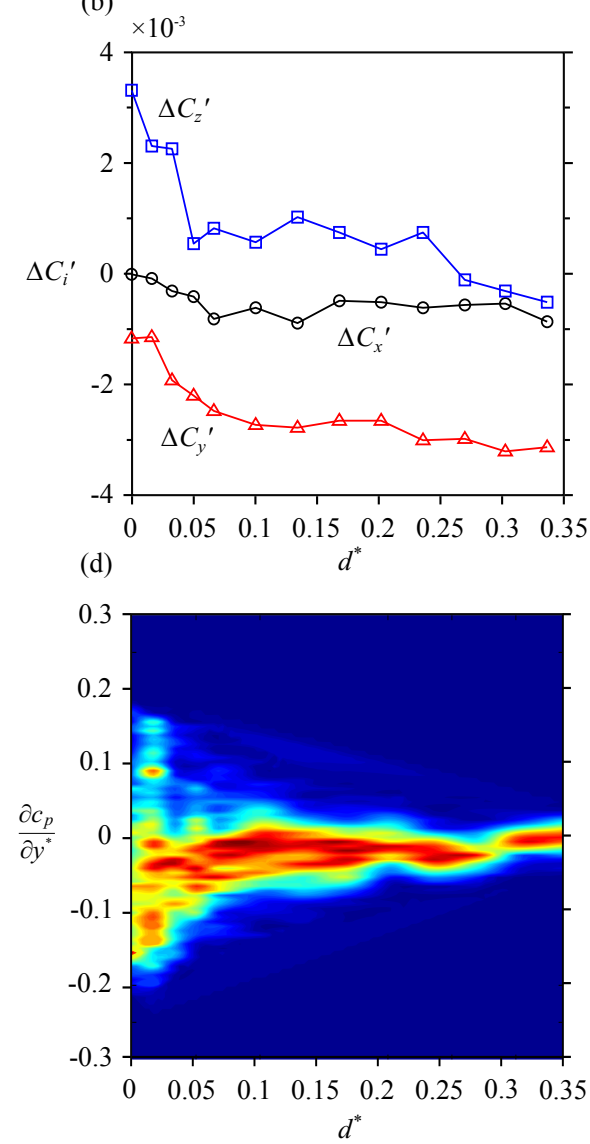

Figure 12: Case with the control cylinder. Variation from the reference case values given in Table 1(see text) of the mean (a) and fluctuation (b) of the force coefficients (empty symbols) as a function of the cavity depth. Variation from the reference case of the mean base suction $C_{b}$ (cross symbols) in (a). Statistics of the vertical (c) and horizontal (d) base pressure gradient as defined in Eq. (3). The PDFs are normalized by their maximum value using the same color bar as in Fig. 2. 

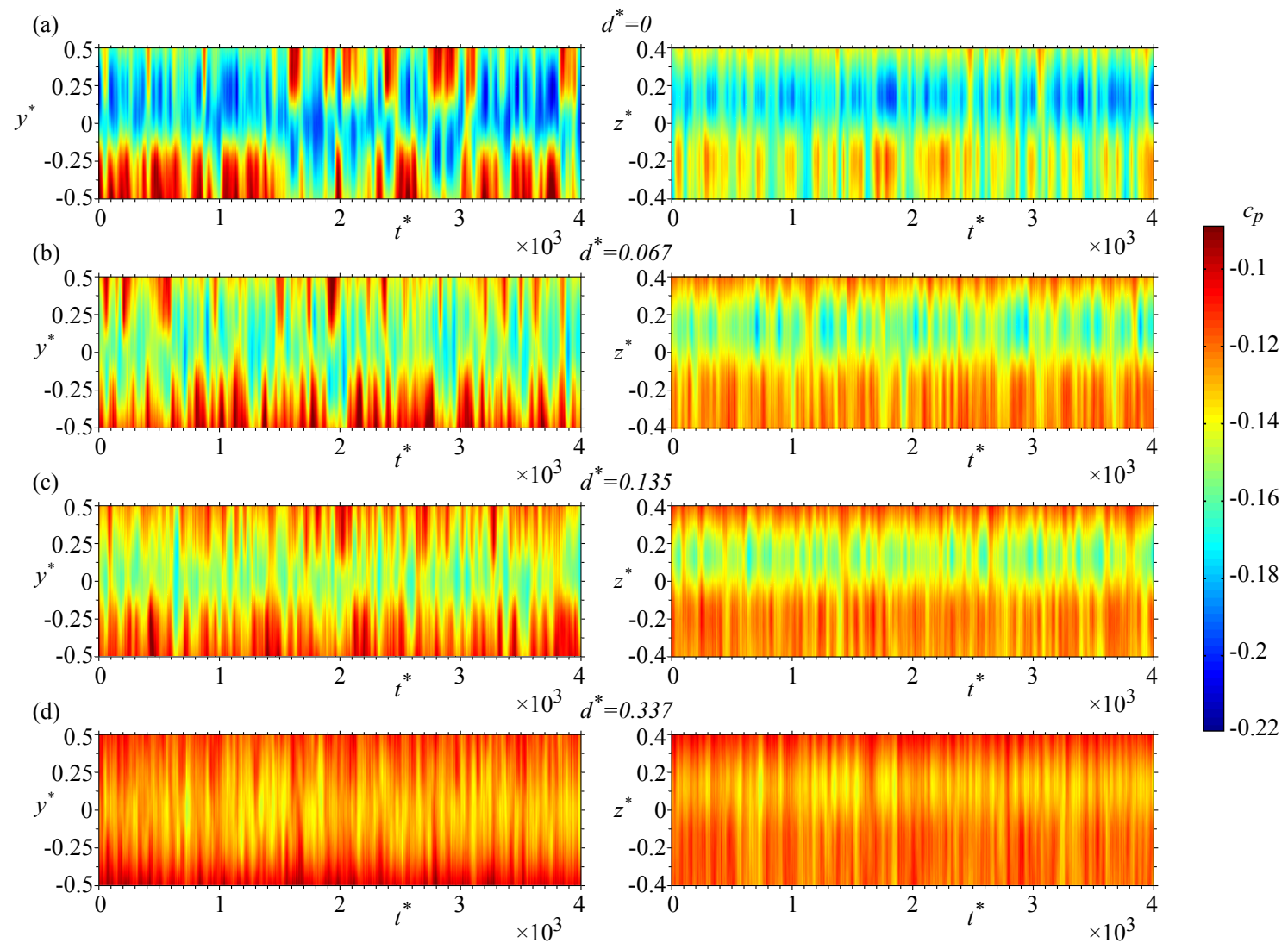

Figure 13: Case with the control cylinder. Space time diagram of the horizontal, $c_{p}\left(y^{*}, t\right)$ (left) and vertical, $c_{p}\left(z^{*}, t\right)$ (right) pressure distribution at the base (Eq. 2) vs. the cavity depth $d^{*}$. 
(a)

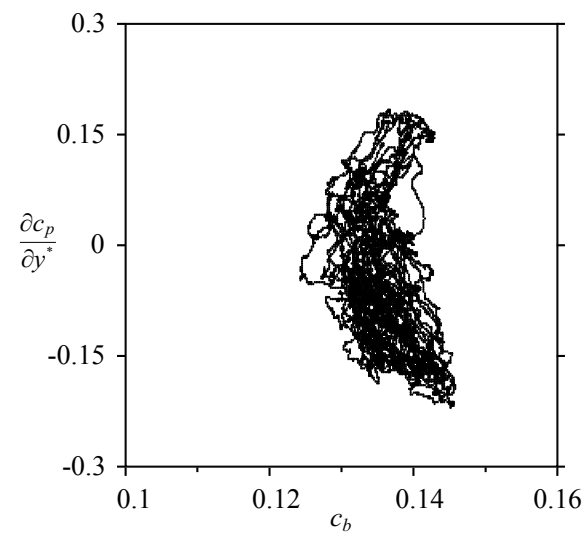

(b)

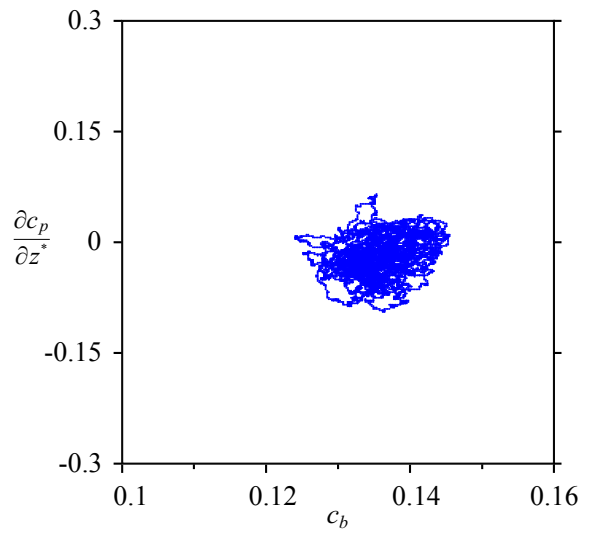

Figure 14: Case with the control cylinder and no cavity $\left(d^{*}=0\right)$. Trajectories in the phase diagram : horizontal base pressure gradient vs. base suction (a) and vertical base pressure gradient vs. base suction (b).

In Fig. 12(a), the drag coefficient and the base suction decrease continuously as the cavity is deepen from $d^{*}=0$ to $d^{*} \sim 0.1$ and then reach a plateau. The smaller magnitude of the drag coefficient variation than the base suction variation indicates a positive contribution of the control cylinder to the overall drag. We can see from the figure that the cylinder contribution to the drag increases as the cavity gets deeper. This effect, that was also observed for another set of experiments at a larger main flow velocity, cannot be deduced from the wake modifications due the cavity only that are shown in Fig. 8 and Fig. 7. From these figures, we see that the cavity effect is to increase the size of the back flow region as well as its intensity at the virtual location of the cylinder compared to the reference case. One would then expect the cylinder to have a negative drag, thus having a negative contribution to the overall drag. The contrary is observed in Fig. 12(a), indicating that the cylinder must modify the back flow consequently.

We can see in Fig. 13 that the base suction decrease as the cavity gets deeper is associated with the disappearance of the low pressure region at the base. As for the cavity effect without the control cylinder, the level of high pressure remains rather unaffected. 


\section{Discussions}

\subsection{Bifurcation theory}

A bifurcated diagram is obtained in Fig. 9(d), with the most probable horizontal pressure gradient as the control parameter and the cavity depth as the order parameter. There is a pitchfork point at $d_{c}^{*} \sim 0.24$, one unique branch for $d^{*}>d_{c}^{*}$ and two branches for $d^{*}<d_{c}^{*}$. For seek of homogeneity with other works it is convenient to adopt as order parameter the negative distance from the threshold $\epsilon=d_{c}^{*}-d^{*}$. In that case a negative $\epsilon$ corresponds to the unique solution branch, and positive epsilon to the bifurcated branches. Note that for positive values, $\epsilon$ is bounded by $d_{c}^{*}$ where the two opposite pressure gradient worth approximately \pm 0.15 (see Fig. 15). The normal form of the bifurcation can be modelled as :

$$
\dot{A}=\epsilon A-\delta A^{3} \equiv-\frac{d V}{d A}
$$

where $A$ is the horizontal base pressure gradient, $\delta=\frac{d_{c}^{*}}{0.15^{2}}$ and $V$ the corresponding potential. The steady solutions of Eq. $5(\dot{A}=0)$ are superimposed to the statistics in Fig. 9(d) (dashed white lines) with a slight negative vertical shift to take into account the symmetry imperfection of our experimental flow geometry. Due to the large Reynolds number of the flow, the system is continuously excited by turbulent fluctuations. Whatever their origins are (free stream turbulent intensity, laminar separation and turbulent reattachment on the forebody, unsteady separation on the support, wake dynamics...), they have to be introduced in Eq. 5 with an additional source term :

$$
\dot{A}=\epsilon A-\delta A^{3}+a(\epsilon) f(t)
$$

which is a Langevin equation where $f$ is a random process and $a$ is its amplitude. In the bifurcated states, the probability of changing states is given by (Gammaitoni et al., 1998):

$$
N_{s} \sim \exp (-\gamma(\epsilon))
$$

where $\gamma$ is the ratio between the potential barrier $\Delta V=\frac{\epsilon^{2}}{4 \delta}$ separating the two stable solutions in Eq. 5 and the kinetic energy of the turbulent fluctuation $\sigma$ playing a role of temperature:

$$
\gamma=\frac{\Delta V}{\sigma}
$$




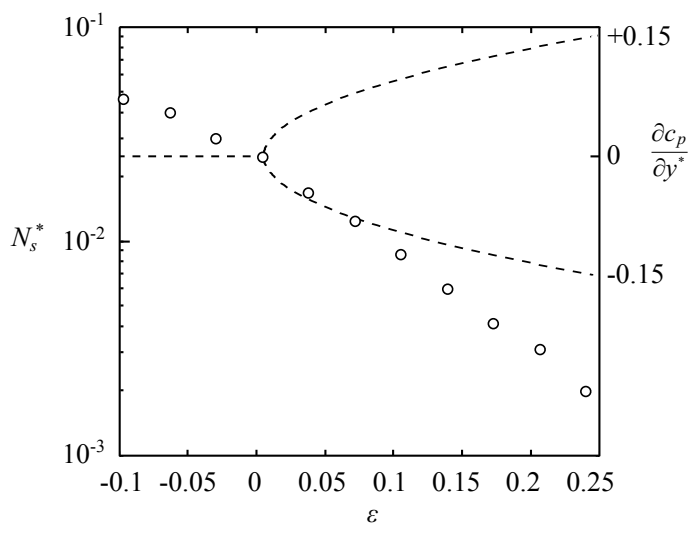

Figure 15: Number of mode switching (empty circles) per unit of dimensionless time $N_{s}^{*}$ as a function of $\epsilon=d_{c}^{*}-d^{*}$. The dashed lines report the pitchfork bifurcation of the horizontal base pressure gradient observed in Fig. 9(d).

The probability of changing states can be computed from pressure time series, we just count the number of time the horizontal base pressure gradient crosses the value 0 indicating a mode switch. Looking at Fig. 3 (left), we can see that the number of switches clearly decreases as the cavity gets shallow, i.e. $\epsilon$ increases. It means that the probability of changing states is a decreasing function of $\epsilon$. It is in agreement with the model, the potential barrier $\Delta V$ becomes larger as $\epsilon$ increases, which renders more difficult for the system to jump from one stable position to the other. We plot in Fig.15 the number of switches per unit of dimensionless time, $N_{s}^{*}$ in a semi-log plot as a function of $\epsilon$. The variation of $\gamma(\epsilon)$ is not fully quadratic as expected by the potential barrier of the model, but rather affine for positive values of $\epsilon$. It might be ascribed to a turbulent fluctuation evolution with $\epsilon$, although the Reynolds number is constant. This effect is possible because the intensity of turbulent fluctuations (see Fig.7d and Fig.8d) was found to be increased in the wake when $\epsilon$ is increased (i.e. cavity is reduced).

\subsection{Mechanism of drag reduction with a body cavity}

The presence of the cavity of depth $d^{*}=0.27$ at the body base is able to achieve a substantial base suction reduction of $18 \%$ compared to the blunt base $\left(d^{*}=0\right)$. The magnitude of the effect is in agreement with previous works about axisymmetric bodies as reported by Viswanath (1996). In our case the base pressure modification produces a net drag reduction of $9 \%$ without changing the condition of flow separation at the rectangular trailing 
edge. The origin is revealed in Fig. 3 and Fig. 6 showing the disappearance of the low pressure region ascribed to the interaction between the rectangular base and the vortex system depicted in Fig. 11(a). As for the interpretation of the cavity effect in 2D flows (Molezzi and Dutton, 1995), it can be argued that the increased distance due to the body cavity from the base to the static vortex system of the RSB mode is the origin of the drag reduction. This mechanism is likely to be generalized to axisymmetric bodies since RSB modes has been evidenced very recently for this geometry (Rigas et al., 2014; Grandemange et al., 2014a). If this is the case, the gain in drag using a body cavity of 3D bluff bodies is directly related to the strength of their RSB modes and substantial gain indicates that RSB modes are major contributors to base suction.

The continuous reduction of the side force fluctuations $C_{y}^{\prime}$ in Fig. 9(b) as the cavity gets deeper is associated with the extinguishment of the bimodal behavior of the side force as can be seen in the statistics in Fig. 16. The side force statistics has indeed the same trend than the horizontal base pressure gradient statistics shown in Fig. 9(d). However these results alone are not sufficient to argue that the RSB mode vortex system is weakened or suppressed by the cavity. For a given strength of a vortex system, both the distance and the additional inner wall introduced by the cavity depth may explain respectively the base pressure gradient reduction and the side force reduction. The decisive arguments showing the weakening and the suppression of the RSB mode wake are the increase of the switching probability as discussed in the previous discussion and the statistics of the global side velocity of the wake shown in Fig.16, where the most probable side velocity becomes zero for deep cavity in consistency with a symmetry preserved wake.

Finally, we summarize the findings about the mechanisms that led to the spectacular drag reduction associated with the cavity depth in Fig. 9(a). For small cavity depths, say $d^{*}<0.05$, the RSB modes strength remains almost unaffected while a large variation of drag is measured. It is then the increased distance between the separation location and the base which reduces the interaction of the $3 \mathrm{D}$ vortex system of the RSB mode with the wall. This lower interaction provokes a raising of the base pressure. This is similar to the drag reduction mechanism of the cavity effect with $2 \mathrm{D}$ bluff bodies (Molezzi and Dutton, 1995). The 2D periodic Kármán vortices are playing the same role as the 3D static vortex system of the RSB mode. For deeper cavities $\left(d^{*}>0.05\right)$, the stabilization process toward the symmetry preserved mode might also contribute to an additional drag reduction with 


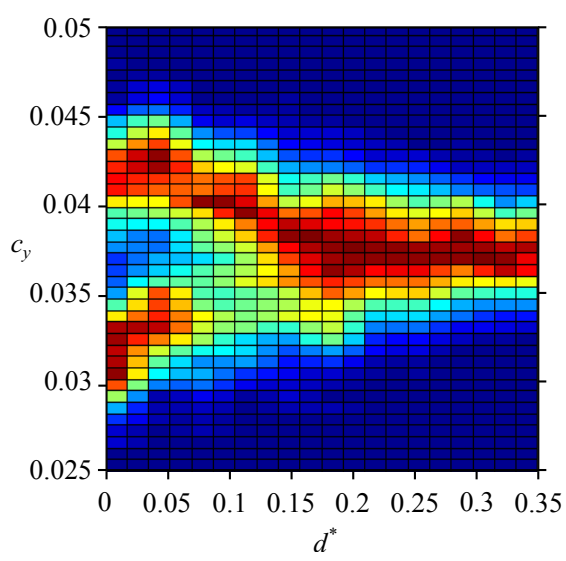

Figure 16: Statistics of the side force coefficient $c_{y}$ as a function of the cavity depth $d^{*}$. The PDFs are normalized by their maximum value using the same color bar as in Fig. 2.

the gradual suppression of the 3D vortex system that is replaced by an elliptical toric recirculation. Once the wake is stabilized $\left(d^{*}>0.24\right)$, the base suction saturates to a constant value but the drag slightly increases due to the additional friction on the inner wall of the cavity.

\section{Conclusion}

The presence of a cavity at the base of the squareback Ahmed model is able to reduce substantially the drag coefficient, while the flow at the separation is not modified. This cavity effect is due to the permanent presence in the natural wake of an energetic 3D static vortex system close to the base of the body (RSB modes). Due to the reflectional symmetry of the rectangular base, there are two RSB mirror modes present in the natural wake that switch from one to the other randomly. It is shown that the cavity is able to stabilize the natural wake toward a symmetry preserved wake, thus suppressing the RSB modes and leading to an elliptical toric recirculation. The stabilization can be modelled with a Langevin equation. The plausible mechanism for drag reduction is based on the interaction of the static $3 \mathrm{D}$ vortex system of the RSB modes with the base of the body and their suppression by stabilization.

It would be interesting to know whether these results apply for axisymmetric bodies with cavity in the turbulent regime. Is the drag reduction observed in these cases (Viswanath, 1996) only related to a simple reduced 
interaction of the SB modes with the body base or associated with a stabilization toward a symmetry preserved mode as in the present case ?

It is shown that the technique of the control cylinder in the recirculating region only affects partially the RSB mode. It also leads to less drag reduction than the cavity effect that suppresses the RSB modes.

\section{Acknowledgments}

Olivier Cadot is very thankful to Luc Pastur for fruitful discussions about bifurcation theory and Langevin equation. The authors wish to thank the CNRT R2A, National Centre for Technologic Research for Aerodynamics and Aeroacoustics of ground vehicles for providing the $2 / 5$ scale wind tunnel of GIE S2A as well as the financial support for the postdoc position of Antoine Evrard. They are grateful to Tony Ruiz and Fabien Harambat for their help during the experiments and thankful to Eric Van Grevenynghe for the PIV acquisitions and treatments.

\section{References}

Ahmed, S., Ramm, G., Faitin, G., 1984. Some salient features of the timeaveraged ground vehicle wake. SAE Technical Paper Series 840300.

Berger, E., Scholz, D., Schumm, M., 1990. Coherent vortex structures in the wake of a sphere and a circular disk at rest and under forced vibrations. Journal of Fluids and Structures 4 (3), 231-257.

Bohorquez, P., Sanmiguel-Rojas, E., Sevilla, A., Jiménez-González, J., Martínez-Bazán, C., 2011. Stability and dynamics of the laminar wake past a slender blunt-based axisymmetric body. Journal of Fluid Mechanics 676 (1), 110-144.

Cadot, O., Evrard, A., Pastur, L., 2015. Imperfect supercritical bifurcation in a three-dimensional turbulent wake. Physical Review E 91 (6).

Choi, H., Jeon, W.-P., Kim, J., 2008. Control of flow over a bluff body. Annual Review of Fluid Mechanics 40, 113-139.

Fabre, D., Auguste, F., Magnaudet, J., 2008. Bifurcations and symmetry breaking in the wake of axisymmetric bodies. Physics of Fluids 20, 051702. 
Gammaitoni, L., Hanggi, P., Jung, P., Marchesoni, F., 1998. Stochastic resonance. Reviews of Modern Physics 70 (1), 223-287.

Grandemange, M., Cadot, O., Courbois, A., Herbert, V., Ricot, D., Ruiz, T., Vigneron, R., 2015. A study of wake effects on the drag of the Ahmed squareback model at the industrial scale. Journal of Wind Engineering and Industrial Aerodynamics 145, 282-291.

Grandemange, M., Gohlke, M., Cadot, O., 2012. Reflectional symmetry breaking of the separated flow over three-dimensional bluff bodies. Physical Review E 86, 035302.

Grandemange, M., Gohlke, M., Cadot, O., 2013a. Bi-stability in the turbulent wake past parallelepiped bodies with various aspect ratios and wall effects. Physics of Fluids 25, 095103.

Grandemange, M., Gohlke, M., Cadot, O., 2013b. Turbulent wake past a three-dimensional blunt body. Part 1. Global modes and bi-stability. Journal of Fluid Mechanics 722, 51-84.

Grandemange, M., Gohlke, M., Cadot, O., 2014a. Statistical axisymmetry of the turbulent sphere wake. Experiments in fluids 55 (11), 1-10.

Grandemange, M., Gohlke, M., Cadot, O., 2014b. Turbulent wake past a three-dimensional blunt body. part 2. experimental sensitivity analysis. Journal of Fluid Mechanics 752, 439-461.

Irving Brown, Y., Windsor, S., Gaylard, A., 2010. The effect of base bleed and rear cavities on the drag of an SUV. SAE Technical Paper Series 201001-0512.

Kruiswyk, R., Dutton, J., 1990. Effects of a base cavity on subsonic nearwake flow. AIAA Journal 28 (11), 1885-1893.

Martin-Alcantara, A., Sanmiguel-Rojas, E., Gutierrez-Montes, C., MartinezBazan, C., 2014. Drag reduction induced by the addition of a multi-cavity at the base of a bluff body. Journal of Fluids and Structures 48, 347-361.

Mittal, R., Wilson, J., Najjar, F., 2002. Symmetry properties of the transitional sphere wake. AIAA journal 40 (3), 579-582. 
Molezzi, M., Dutton, J., 1995. Study of subsonic base cavity flowfield structure using particle image velocimetry. AIAA Journal 33 (2), 201-209.

Morel, T., 1979. Effect of base cavities on the aerodynamic drag of an axisymmetric cylinder. Aeronautical Quarterly 30, 400-412.

Pasquetti, R., Peres, N., 2015. A penalty model of synthetic micro-jet actuator with application to the control of wake flows. Computers \& Fluids $114(0), 203-217$.

Pier, B., 2008. Local and global instabilities in the wake of a sphere. Journal of Fluid Mechanics 603, 39-61.

Rigas, G., Oxlade, A., Morgans, A., Morrison, J., 2014. Low-dimensional dynamics of a turbulent axisymmetric wake. Journal of Fluid Mechanics $755,159$.

Roshko, A., 1993. Perspectives on bluff body aerodynamics. Journal of Wind Engineering and Industrial Aerodynamics 49 (1-3), 79-100.

Sanmiguel-Rojas, E., Jimenez-Gonzalez, J. I., Bohorquez, P., Pawlak, G., Martinez-Bazan, C., 2011. Effect of base cavities on the stability of the wake behind slender blunt-based axisymmetric bodies. Physics of Fluids 23 (11), 114103.

Schlichting, H., Gersten, K., 2000. Boundary layer theory. Springer Verlag.

Strykowski, P., Sreenivasan, K., 1990. On the formation and suppression of vortex shedding at low Reynolds numbers. Journal of Fluid Mechanics 218, $71-107$.

Viswanath, P., 1996. Flow management techniques for base and afterbody drag reduction. Progress in Aerospace Sciences 32 (2-3), 79-129.

Waudby-Smith, P., Bender, T., Vigneron, R., 2004. The GIE S2A full-scale aeroacoustic wind tunnel. SAE Technical Paper Series 2004-01-0808.

Wu, T., 1972. Cavity and wake flows. Annual Review of Fluid Mechanics 4 (1), 243-284. 